\title{
Educational Intervention Improves Proton Pump Inhibitor Stewardship in Outpatient Gastroenterology Clinics
}

\author{
Megan J. Walkera, Nicholas R. Crews ${ }^{\mathrm{a}}$, Mustapha El-Halabi ${ }^{\mathrm{a}}$, Nabil F. Fayad ${ }^{\mathrm{a}}$, b
}

\begin{abstract}
Background: Improper chronic proton pump inhibitor (PPI) use has risen significantly in the last few decades. In our gastroenterology trainees' clinics, we aimed to optimize PPI usage.

Methods: We collected baseline data on patients' PPI use for 8 weeks. Based on gastroenterology society guidelines, we determined conditions for appropriate PPI use. If the indication could not be determined, it was categorized as "unknown". Generated from the three most frequent causes for inappropriate PPI use, interventions were developed to correct each issue. Following a brief educational session, trainees implemented these interventions over a subsequent 8 -week interval.
\end{abstract}

Results: During our pre-intervention period, trainees evaluated 263 patients who were prescribed a PPI. In $49 \%$ of the cases, the use of PPI was deemed inappropriate. The most common reasons were: gastroesophageal reflux disease (GERD) which was never titrated to the lowest effective dose, twice daily dosing for Barrett's esophagus (BE) chemoprevention and unknown indication. During our intervention period, trainees evaluated 145 patients prescribed a PPI for GERD with well-controlled symptoms in 101 cases. PPI had not been titrated to lowest effective dose in 37 cases prompting intervention which was successful in 23 cases. PPI indication was unknown in 17 cases prompting a message to the prescribing provider to review appropriateness. Two cases of BE chemoprevention with twice daily dosing were appropriately reduced to daily dosing. Ultimately, after intervention, PPI use was deemed appropriate after intervention in $172(77 \%)$ cases.

Conclusions: Improper chronic PPI use was significant. Focusing intervention efforts on PPI use for GERD, BE and unknown indications substantially increased appropriateness of PPI use.

Keywords: Stewardship; Prescribing initiatives; Appropriate use; Proton pump inhibitors

Manuscript submitted October 24, 2019, accepted November 5, 2019

a Division of Gastroenterology and Hepatology, Indiana University School of Medicine, 702 Rotary Circle, Suite 225, Indianapolis, IN 46202, USA

${ }^{b}$ Corresponding Author: Nabil F. Fayad, Section of Gastroenterology and Hepatology, Richard L. Roudebush VA Medical Center, 1481 W 10th St, Indianapolis, IN 46202, USA. Email: nfayad@iu.edu

doi: https://doi.org/10.14740/gr1238

\section{Introduction}

Proton pump inhibitors (PPIs) have been widely prescribed and heavily marketed since their introduction in 1989. Excellent short-term efficacy of PPI therapy has been demonstrated for specific indications including erosive esophagitis and upper gastrointestinal (GI) bleeding due to peptic ulcer disease (PUD) [1-3]. Chronic use of PPI therapy is frequently prescribed for chemoprevention of acid-related complications and ulcer disease in addition to maintenance therapy for chronic conditions including dyspepsia, eosinophilic esophagitis (EoE) and gastroesophageal reflux disease (GERD) $[4,5]$.

However, improper chronic PPI use has risen significantly in the last few decades with PPI misuse reported to range from $20 \%$ to $80 \%$ across both hospital and ambulatory settings $[6,7]$. Though rates were lower among academic institutions compared to non-academic, they are still at nearly $50 \%$ [8]. The most frequently cited reasons for PPI overuse include the prevention of peptic ulcers in patients without risk factors, stress ulcer prophylaxis in non-intensive care units and the overtreatment of functional dyspepsia $[9,10]$.

PPIs have favorable safety profiles in short-term use with most common side effects of headache and diarrhea occurring in $2-5 \%$ of patients. However, the safety of chronic PPI use has been challenged recently in highly publicized reports noting associations with chronic kidney disease, dementia and bone fractures [11-14]. Overall usage of PPI decreased in 2012 for the first time since its introduction in 1989 which may be related to the rising fears of potential PPI-related side effects [15].

PPI stewardship programs are being developed in which active PPI prescription appropriateness is reassessed. Pharmacy-driven initiatives have focused on deprescribing inappropriate PPI treatments in long-term care facilities' patients and in geriatric patients at risk of polypharmacy [16-18]. We recognize that our institution's providers and our patients are not immune to this issue. Thus, we developed a quality improvement to optimize PPI use at our institution. The purpose of this quality improvement project is to optimize our patients' usage of PPIs and thus decrease their risk for potential adverse events from inappropriate chronic PPI use. We aimed to ensure that our patients were prescribed PPIs for an appropriate indication, especially in cases of chronic ( $>12$ weeks) PPI use.

\section{Materials and Methods}

The quality improvement study was developed and executed 
by the trainees in our gastroenterology and hepatology fellowship program within the context of our trainee continuity clinics at the affiliated county hospital and veterans affairs (VA) medical center. The project leaders were the authors of this manuscript: one GI fellow team leader at each of the two clinics, the chief fellow and the GI fellowship program director. All trainees who participated in the initiative were gastroenterology fellows. As the project was designed as a quality improvement initiative, this study was exempt from institutional review board (IRB) review. This study was conducted in compliance with the ethical standards of the responsible institution.

To first determine the extent of inappropriate PPI use in the trainee continuity clinics and evaluate the most frequent causes, baseline data were collected during an 8-week time period. During this pre-intervention phase, only data pertaining to patients' medications prior to the time of the clinic visit were captured to avoid bias. Any changes made during the clinic visit were not reflected in this initial data collection. During each encounter, the use of PPI was documented (including drug name, dose, frequency, duration and indication) in a designated location in the clinical note. The use of other antacids, including $\mathrm{H}_{2}$ blockers, was not collected during this study as this was not the focus of this quality initiative. Basic demographic information, including age and sex, was collected from each patient. Based upon approved indications for PPIs published in the gastroenterology society guidelines, indications were noted as predetermined categories: GERD, dyspepsia, PUD, Barrett's esophagitis (BE), EoE and Helicobacter pylori [19-26]. For each patient, all applicable indications were captured. If the apparent indication for PPI use was not consistent with any of the above, it was categorized as "other". If the indication was neither known by the patient nor apparent upon review of the two most recent primary care physicians' and GI physicians' notes in the medical record, it was designated as "unknown".

At the end of this 8-week pre-intervention phase, a retrospective examination of each trainee's patient encounters was performed by another trainee to determine whether PPI use had been appropriate or inappropriate in each case. Appropriateness was assessed by the aid of an algorithmic flowchart created by the project leaders. The flowchart included guideline-based recommendation statements describing appropriate PPI use for the various upper GI conditions noted above. The algorithmic flowchart guided trainees by using a series of yes or no questions based upon indication for PPI prescription. If the medication was prescribed for more than one indication, the algorithm guided trainees to follow the pathway for the indication with the most lenient PPI prescribing guidelines (GERD in the majority of cases). In some cases, additional information was abstracted from the medical record to make this determination. For example, in patients with PUD due to a nonsteroidal anti-inflammatory drug (NSAID) who remained on PPI therapy after the initial 8-week treatment period, continuation was considered appropriate if the patient required to remain on the offending NSAID. In patients with GERD, history of reflux esophagitis, presence of ongoing symptoms and whether previous dose titration had been attempted were recorded. In patients with dyspepsia and PUD, prior $H$. pylori testing status was noted as this would affect therapy accept- ability under guidelines. For all patients with PUD, the ulcer etiology was captured (H. pylori, medication-related, hypersecretory conditions, idiopathic) as acceptability of continued PPI use varies depending on etiology. If PUD was medicationrelated, the reviewers determined if the offending medication was continued or discontinued after ulcer disease was diagnosed. PPI use automatically was deemed inappropriate if the indication was either "other" or "unknown". The most frequent noted indication in the "other" category was use of PPI in the setting of esophageal varices with or without a history of prior variceal bleeding. Finally, the project leaders compiled and analyzed the data.

To achieve the greatest impact, the intervention was directed towards reducing the inappropriate PPI use for the three most common conditions associated with PPI misuse as identified during the pre-intervention phase. The intervention included an educational session and a corresponding template with a stepwise approach to be utilized when completing clinical documentation. The education session consisted of a brief PowerPoint presentation describing the approved indications for PPIs with discussion of appropriate and inappropriate use [9]. When only prescribed for GERD, the education emphasized the importance of titrating PPI to the lowest effective dose. The presentation also underscored that only once daily dosing is recommended as maintenance PPI therapy for nonGERD conditions. During the educational session, the providers were given an algorithm similar to that used in the preintervention phase to illustrate appropriate PPI use and were also offered additional guidance to correct misuse for the three main indications identified (Fig. 1).

During the 8-week intervention period, the providers completed the template integrated into the clinical documentation. The template noted a series of steps, the first of which was a reminder to perform a GI medication reconciliation with the patient, especially focusing on PPIs. The indication was again noted for each patient with specific reminders in the template addressing GERD, BE and unknown indications. If the indication was discovered to be unknown, a message was sent in the electronic medical record to the patient's primary care physician to inquire if the drug should be continued. For indications of GERD and BE, the template included reminders of the appropriate use of PPI in these settings and prompted providers to modify the drug dosing during the visit if appropriate and document the changes. For indications other than unknown, GERD and BE, any changes to PPI therapy were up the discretion of the provider.

At the conclusion of the 8-week intervention phase, the clinical documentation was reviewed by another trainee who determined if PPI use had been appropriate in each case. The data were again compiled and analyzed by the project leaders.

\section{Results}

During the 8-week pre-intervention phase, 566 patients were evaluated by 15 trainee providers in the county hospital and associated VA gastroenterology clinics. Of those 566 patients, $263(46 \%)$ patients were prescribed a PPI. Esomeprazole, omeprazole and pantoprazole were the prescribed PPIs in 260 


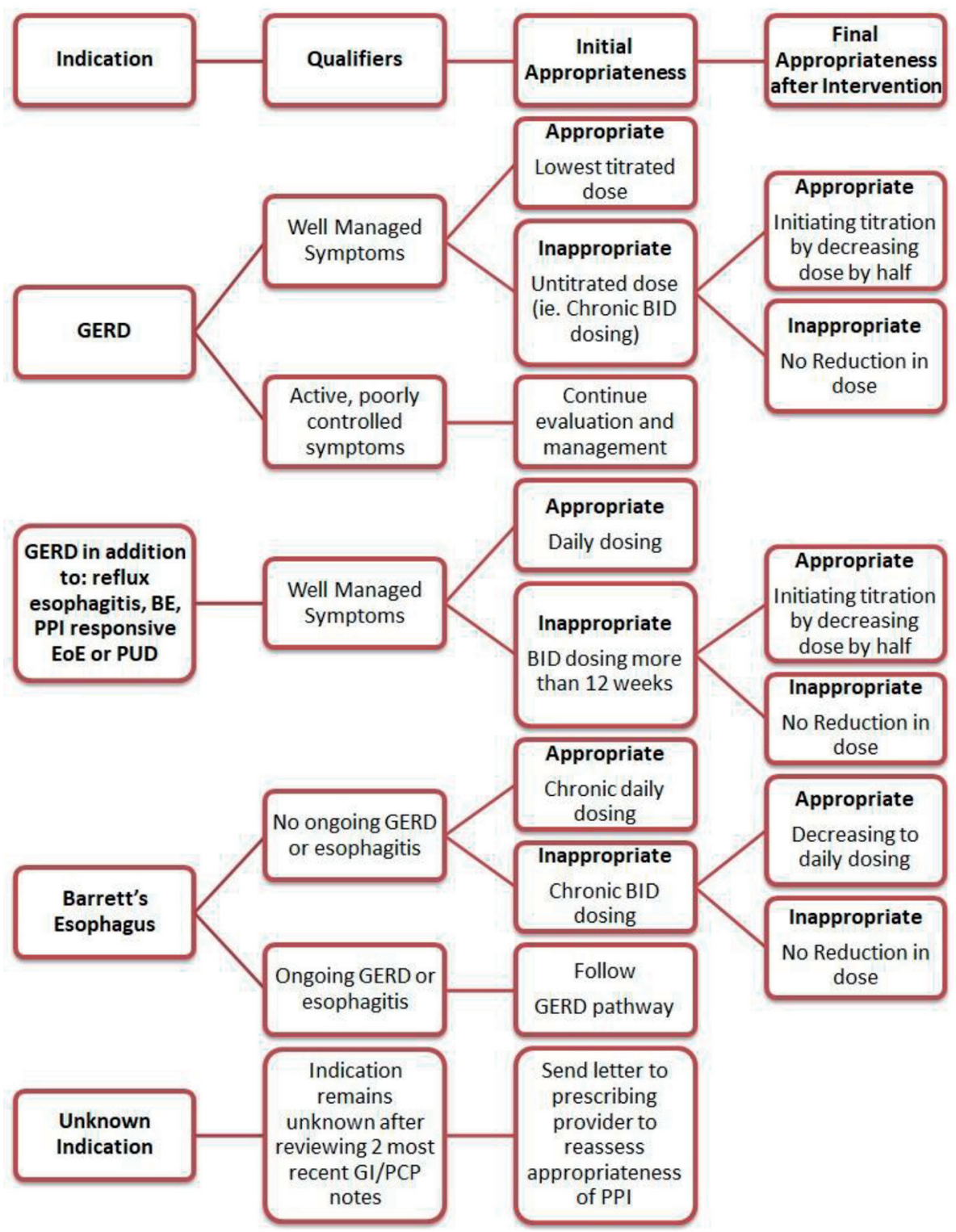

Figure 1. Algorithm of interventions based on PPI indication. PPI: proton pump inhibitor; GERD: gastroesophageal reflux disease; BE: Barrett's esophagus; EoE: eosinophilic esophagitis; PUD: peptic ulcer disease; PCP: primary care provider; GI: gastrointestinal.

of the 263 cases. Among those 263 patients, the most common indication was GERD (64\%), followed by dyspepsia (10\%), BE chemoprevention (8\%) and PUD (5\%). The "other" category included $13(5 \%)$ patients which included three cases of PUD prophylaxis for chronic steroid use, four cases of variceal bleeding prophylaxis and four cases of globus sensation or laryngopharyngeal symptoms. After our reviewers assessed PPI appropriateness employing the guideline-based algorithm, PPI use was found to be inappropriate in 129 patients $(49 \%$ of patients prescribed a PPI). The most frequent cause for PPI misuse (found in 64 patients $(50 \%)$ of patients with inappropriate PPI use) was PPI taken for an indication of GERD alone which was never titrated to lowest effective dose. Of these 64 cases, 42 patients were on a double dose PPI. The second most com- mon inappropriate use was an "unknown" indication for the drug ( $14 \%$ of patients with inappropriate PPI use). The third most common inappropriate use was continued use of double dose PPI for BE chemoprevention while patient did not have any active GERD symptoms ( $10 \%$ of patients with inappropriate PPI use). Combined, these three categories of inappropriate PPI use accounted for nearly $75 \%$ of cases of PPI misuse. Table 1 reports all PPI indications and appropriateness of use.

During the 8-week intervention phase, $224(46 \%)$ of 482 patients seen in our gastroenterology clinics were actively taking a PPI prior to clinic visit. PPI use was deemed appropriate without intervention in $130(58 \%)$ of 224 patients prescribed a PPI. A successful intervention was performed in 42 cases, thus ultimately increasing our appropriate PPI use to 172 (77\%) of 


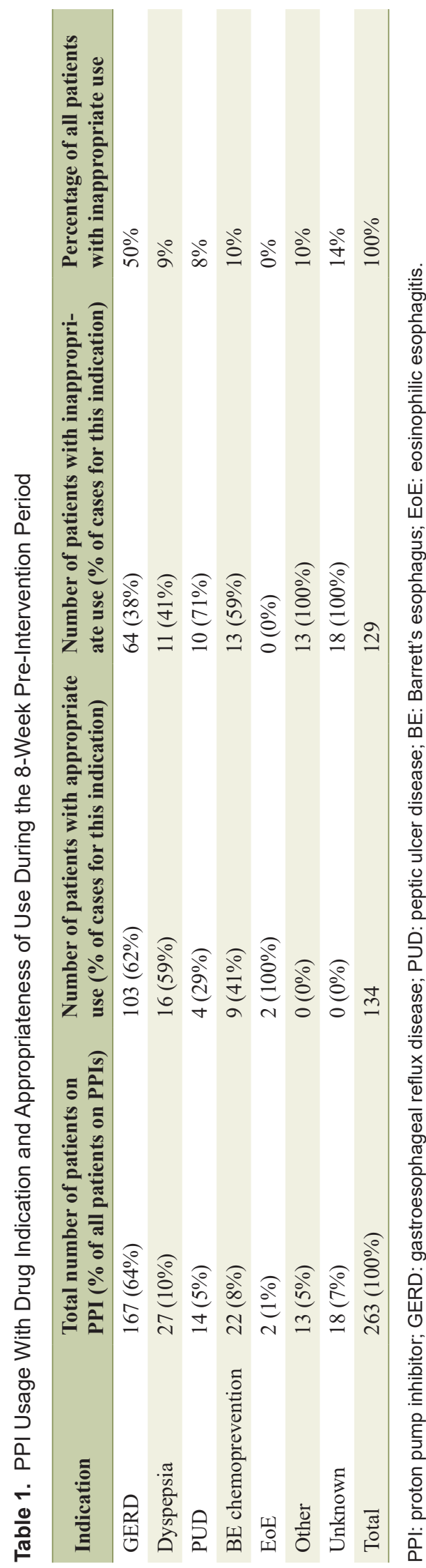

224 cases after intervention (Table 2). Details of our focused interventions for our three indications of interest are described below.

At the time of clinic visit, GERD symptoms were well controlled in $101(70 \%)$ of the 145 patients prescribed a PPI for GERD. A provider had previously titrated the PPI to the lowest effective dose in 64 of the 101 well-controlled patients: standard dosing in 61 patients and half dose daily or as needed (PRN) dosing in three patients. However, 37 of the 101 (37\%) patients were on double dose PPI therapy but had not attempted to titrate down to lowest effective dosing. These cases were deemed to be inappropriate PPI use and the providers successfully decreased the PPI dose by at least half in $23(62 \%)$ of the 37 cases. The most common cause cited for failed attempt to de-escalate the dose was the patient's unwillingness to reduce the dose due to concerns about increased frequency of uncontrolled reflux symptoms.

The indication for PPI therapy was BE chemoprevention in $15(7 \%)$ of 224 patients prescribed PPI who were evaluated during the 8-week intervention phase. Two cases of $\mathrm{BE}$ chemoprevention had been inappropriately maintained on BID dosing and this was appropriately reduced to daily dosing. The other 13 cases were already appropriately dosed and did not require further dosing modification.

The indication of PPI was unknown in $17(8 \%)$ cases after discussion with the patient and review of the electronic medical record (EMR). This finding prompted the provider to send a message to the prescribing provider to review appropriateness and this was completed in all 17 cases.

Despite our focused interventions, 52 cases were considered having inappropriate PPI use at the time of analysis. These 52 cases included 14 patients who refused the provider's intervention because of concern about worsening GERD symptoms if PPI regimen was adjusted, 24 patients with inappropriate PPI use prescribed for an indication that was not specifically addressed by the intervention and 14 cases during which the provider had to address more urgent issues and could not determine nor discuss PPI appropriateness in the allotted visit time.

\section{Discussion}

In our study, significant PPI misuse was discovered in a population of ambulatory gastroenterology patients seen in trainee continuity clinics in VA and county hospital settings. Baseline data established that the most common cause of inappropriate use was maintenance non-titrated double dose PPI use in patients with uncomplicated GERD who had achieved control of their symptoms. Other leading causes of misuse were double dosing of PPI for BE chemoprevention and the absence of an apparent indication after review of the EMR and discussion with the patient. An intervention was developed to address these three most common issues. Intervention included a brief educational session and a written template integrated into clinical notes, both centered on an algorithm approach. Inappropriate PPI use decreased to $23 \%$ after intervention compared to $49 \%$ at baseline.

Our results are similar to previous studies assessing PPI 
Table 2. PPI Appropriateness of Use During the 8-Week Intervention Period

\begin{tabular}{|llll}
\hline Indication & $\begin{array}{l}\text { Total number of patients } \\
\text { on PPI due to indication }\end{array}$ & $\begin{array}{l}\text { Number of patients with inappro- } \\
\text { priate use prior to intervention }\end{array}$ & $\begin{array}{l}\text { Number of patients successfully con- } \\
\text { verted to appropriate use by intervention }\end{array}$ \\
\hline Well-controlled GERD & 101 & 37 & 23 \\
BE chemoprevention & 15 & 2 & 2 \\
Unknown & 17 & 17 & 17 \\
\hline
\end{tabular}

PPI: proton pump inhibitor; GERD: gastroesophageal reflux disease; BE: Barrett's esophagus.

misuse in the outpatient setting, including the high prevalence of unknown indication for PPI prescription [7]. One major cause for this phenomenon is assumed to be the continuation of the medication after a hospitalization. A study of this practice reported that two-thirds of discharge summaries indicated that the medication should be continued at home; however, no clear indication to do so could be identified [27]. Furthermore, in up to $70 \%$ of the cases, these medications are continued chronically after discharge by primary care providers (PCPs) [28]. These findings highlight the often noted deficiencies in communication between providers during transitions of care. In an attempt to mitigate the prevalence of unknown indications for PPIs, our intervention focused on thorough medication reconciliation with the patient and a mandate to communicate to PCPs or prescribing providers when discovered. Our project did not include requesting feedback from the PCPs to determine if the inappropriate PPI use was appropriately changed or stopped. This is a limitation which could be pursued in future iterations of this quality improvement project.

Our study found GERD to be the most common diagnosis associated with chronic PPI overuse, though the initial indication may have been appropriate. The studies which established PPIs as the preferred agents for treatment of GERD focused on healing of erosive esophagitis as their primary endpoint. However, it has since been realized that up to $70 \%$ of GERD patients can be categorized as having non-erosive reflux disease (NERD) [29], thus the goal of therapy is symptom control alone. The American Gastroenterology Association guideline on the management of GERD recommends to decrease therapy to the lowest effective dose; however, this is infrequently done $[21,22]$. PPIs are commonly continued, often at high doses, long term without reassessment or dose reduction [7]. Attempts to step down therapy have been identified in only $20-33 \%$ of patients with uncomplicated GERD alone [30].

Excessive dose of PPI for BE chemoprevention was a significant cause of inappropriate PPI prescription in our study. However, this is not a commonly cited issue reported in the literature. In fact, all of the cases of inappropriate PPI use for BE chemoprevention in our study were noted at the VA hospital. This discrepancy was likely due to the increased prevalence of $\mathrm{BE}$ in our VA patient cohort compared to the county hospital cohort. Of note, the aspect trial was published after the start of our quality improvement project [31]. Using a combined endpoint of time to all-cause mortality, esophageal adenocarcinoma, or high-grade dysplasia, this study supported the use of high dose twice daily PPI over single dose daily PPI. As these findings have not been formally integrated into recommen- dations in the gastroenterology society published guidelines, double dose PPI was still considered inappropriate for the purposes of our study. Over-prescription of PPI for dyspepsia was not prevalent in our study, though it is one of the most commonly cited inappropriate indications in the literature. However, dyspepsia was the second most common indication for PPI use noted in our study's baseline data collection. Therefore, the educational interventions described above in relation to GERD can be similarly applied to dyspepsia.

The strengths of our study stem from the intervention itself. Though our study was implemented by trainees at an academic center, the algorithm applied is user-friendly and widely applicable in many practice settings, including primary care, as it is based upon gastroenterology society guidelines. The tool could also be expanded to pharmacists, as has been done in other studies, to help alert providers to possible inappropriate PPI use. One limitation of our study is generalizability, as it was conducted at an academic center in the trainees' general gastroenterology clinics. Our intervention focused on education of trainees to step down therapy when appropriate and appeared to have significant success. Given that our study was conducted over a relatively short time-frame by clinicians who arguably had a vested interest in improving PPI misuse, this could be viewed as another limitation. In a review of 21 studies aiming to discontinue inappropriate PPI use, only six noted effective interventions, one of which was based on provider education [30]. Three of the other studies based on education were ineffective. The authors concluded that clinician enthusiasm was likely a significant factor in potential success of such interventions.

Future areas of improvement for this quality project include performing another Plan-Do-Act cycle with a modified intervention to achieve the goal of $100 \%$ appropriate PPI use for these three specific conditions and eventually additional conditions associated with misuse. Another area for further study would be to investigate the durability of the intervention to determine if the improvement in PPI misuse was maintained after the conclusion of the project. Other future directions include disseminating this algorithmic approach intervention to other GI training programs and also to internal medicine and family medicine training programs to improve appropriate PPI use for primary care physicians.

In conclusion, our study highlights that failure to step down therapy in GERD is a significant and primary cause of inappropriate chronic PPI use. With an education-based intervention using an algorithm adapted from multiple GI society guidelines, PPI misuse can be significantly reduced in GERD and other conditions. 


\section{Acknowledgments}

The authors thank the Indiana University gastroenterology fellows who also participated in the data collection and implementation of this project.

\section{Financial Disclosure}

The authors declare no financial arrangements regarding this manuscript, which was not supported by any grant funding.

\section{Conflict of Interest}

The authors declare no conflict of interest regarding this manuscript.

\section{Author Contributions}

MJW and NRC participated in the design of the project, data collection and analysis, and equally contributed to the writing, editing and preparation of the manuscript. MEH participated in the design of the project, data collection and editing of the manuscript. NFF oversaw the study design process and execution, and contributed to editing of the manuscript.

\section{References}

1. Lau JY, Leung WK, Wu JC, Chan FK, Wong VW, Chiu PW, Lee VW, et al. Omeprazole before endoscopy in patients with gastrointestinal bleeding. N Engl J Med. 2007;356(16):1631-1640.

2. Leontiadis GI, Sharma VK, Howden CW. Proton pump inhibitor therapy for peptic ulcer bleeding: Cochrane collaboration meta-analysis of randomized controlled trials. Mayo Clin Proc. 2007;82(3):286-296.

3. Chiba N, De Gara CJ, Wilkinson JM, Hunt RH. Speed of healing and symptom relief in grade II to IV gastroesophageal reflux disease: a meta-analysis. Gastroenterology. 1997;112(6):1798-1810.

4. Yadlapati R, Kahrilas PJ. When is proton pump inhibitor use appropriate? BMC Med. 2017;15(1):36.

5. Scarpignato C, Gatta L, Zullo A, Blandizzi C, for the SIFAIGO-FIMMG Group \& on behalf of the Italian Society of Pharmacology, the Italian Association of Hospital Gastroenterologists, and the Italian Federation of General Practitioners. Effective and safe proton pump inhibitor therapy in acid-related diseases - A position paper addressing benefits and potential harms of acid suppression. BMC Med. 2016;14(1):179.

6. Rakesh TP. Proton pump inhibitors: use, misuse and concerns about long-term therapy. Clin J Gastroenterol. 2011;4(2):53-59.

7. Boghossian TA, Rashid FJ, Thompson W, Welch V, Moayyedi P, Rojas-Fernandez C, Pottie K, et al. Depre- scribing versus continuation of chronic proton pump inhibitor use in adults. Cochrane Database Syst Rev. 2017;3:CD011969.

8. Eid SM, Boueiz A, Paranji S, Mativo C, Landis R, Abougergi MS. Patterns and predictors of proton pump inhibitor overuse among academic and non-academic hospitalists. Intern Med. 2010;49(23):2561-2568.

9. Savarino V, Marabotto E, Zentilin P, Furnari M, Bodini G, De Maria C, Pellegatta G, et al. Proton pump inhibitors: use and misuse in the clinical setting. Expert Rev Clin Pharmacol. 2018;11(11):1123-1134.

10. Savarino V, Tosetti C, Benedetto E, Compare D, Nardone G. Appropriateness in prescribing PPIs: A position paper of the Italian Society of Gastroenterology (SIGE) - Study section "Digestive Diseases in Primary Care". Dig Liver Dis. 2018;50(9):894-902.

11. Lazarus B, Chen Y, Wilson FP, Sang Y, Chang AR, Coresh J, Grams ME. Proton Pump Inhibitor Use and the Risk of Chronic Kidney Disease. JAMA Intern Med. 2016;176(2):238-246.

12. Targownik LE, Leslie WD, Davison KS, Goltzman D, Jamal SA, Kreiger N, Josse RG, et al. The relationship between proton pump inhibitor use and longitudinal change in bone mineral density: a population-based study [corrected] from the Canadian Multicentre Osteoporosis Study (CaMos). Am J Gastroenterol. 2012;107(9):13611369.

13. Yu EW, Bauer SR, Bain PA, Bauer DC. Proton pump inhibitors and risk of fractures: a meta-analysis of 11 international studies. Am J Med. 2011;124(6):519-526.

14. Gomm W, von Holt K, Thome F, Broich K, Maier W, Fink A, Doblhammer G, et al. Association of proton pump inhibitors with risk of dementia: a pharmacoepidemiological claims data analysis. JAMA Neurol. 2016;73(4):410416.

15. Freedberg DE, Kim LS, Yang YX. The risks and benefits of long-term use of proton pump inhibitors: expert review and best practice advice from the American Gastroenterological Association. Gastroenterology. 2017;152(4):706715 .

16. Farrell B, Black C, Thompson W, McCarthy L, RojasFernandez C, Lochnan H, Shamji S, et al. Deprescribing antihyperglycemic agents in older persons: Evidencebased clinical practice guideline. Can Fam Physician. 2017;63(11):832-843.

17. Doell A, Walus A, To J, Bell A. Quantifying candidacy for deprescribing of proton pump inhibitors among longterm care residents. Can J Hosp Pharm. 2018;71(5):302307.

18. Avraham O, Biglow M. Implementation of proton pump inhibitor deprescription protocol in geriatric residents. Ann Pharmacother. 2018;52(8):747-753.

19. Chey WD, Leontiadis GI, Howden CW, Moss SF. ACG clinical guideline: treatment of helicobacter pylori infection. Am J Gastroenterol. 2017;112(2):212-239.

20. Dellon ES, Gonsalves N, Hirano I, Furuta GT, Liacouras CA, Katzka DA, American College of G. ACG clinical guideline: Evidenced based approach to the diagnosis and management of esophageal eosinophilia and eosinophilic 
esophagitis (EoE). Am J Gastroenterol. 2013;108(5):679692; quiz 693.

21. Kahrilas PJ, Shaheen NJ, Vaezi MF, Hiltz SW, Black E, Modlin IM, Johnson SP, et al. American Gastroenterological Association Medical Position Statement on the management of gastroesophageal reflux disease. Gastroenterology. 2008;135(4):1383-1391, 1391 e1381-1385.

22. Katz PO, Gerson LB, Vela MF. Guidelines for the diagnosis and management of gastroesophageal reflux disease. Am J Gastroenterol. 2013;108(3):308-328; quiz 329.

23. Laine L, Jensen DM. Management of patients with ulcer bleeding. Am J Gastroenterol. 2012;107(3):345-360; quiz 361.

24. Lucendo AJ, Molina-Infante J, Arias A, von Arnim U, Bredenoord AJ, Bussmann C, Amil Dias J, et al. Guidelines on eosinophilic esophagitis: evidence-based statements and recommendations for diagnosis and management in children and adults. United European Gastroenterol J. 2017;5(3):335-358.

25. Moayyedi PM, Lacy BE, Andrews CN, Enns RA, Howden CW, Vakil N. Corrigendum: $\mathrm{ACG}$ and $\mathrm{CAG}$ clinical guideline: management of dyspepsia. Am J Gastroenterol. 2017;112(9):1484.

26. Shaheen NJ, Falk GW, Iyer PG, Gerson LB, American
College of G. ACG clinical guideline: diagnosis and management of Barrett's esophagus. Am J Gastroenterol. 2016;111(1):30-50; quiz 51.

27. Ahrens D, Chenot JF, Behrens G, Grimmsmann T, Kochen MM. Appropriateness of treatment recommendations for PPI in hospital discharge letters. Eur J Clin Pharmacol. 2010;66(12):1265-1271.

28. Parente F, Cucino C, Gallus S, Bargiggia S, Greco S, Pastore L, Bianchi Porro G. Hospital use of acid-suppressive medications and its fall-out on prescribing in general practice: a 1-month survey. Aliment Pharmacol Ther. 2003;17(12):1503-1506.

29. Savarino V, Dulbecco P, de Bortoli N, Ottonello A, Savarino E. The appropriate use of proton pump inhibitors (PPIs): Need for a reappraisal. Eur J Intern Med. 2017;37:19-24.

30. Wilsdon TD, Hendrix I, Thynne TR, Mangoni AA. Effectiveness of interventions to deprescribe inappropriate proton pump inhibitors in older adults. Drugs Aging. 2017;34(4):265-287.

31. Jankowski JAZ, de Caestecker J, Love SB, Reilly G, Watson P, Sanders S, Ang Y, et al. Esomeprazole and aspirin in Barrett's oesophagus (AspECT): a randomised factorial trial. Lancet. 2018;392(10145):400-408. 